\title{
Melting of dusty plasma crystals in oscillating external fields
}

\author{
G P Hoffmann and H Löwen \\ Institut für Theoretische Physik II, Heinrich-Heine-Universität Düsseldorf, Universitätsstraße 1, \\ D-40225 Düsseldorf, Germany
}

Received 1 June 2000, in final form 20 July 2000

\begin{abstract}
The melting transition of a charge-polydisperse dusty plasma crystal under the influence of a time-dependent oscillating field is studied within a simple model. The stochastic dynamics of the dust particles is modelled by a Fokker-Planck description. Using non-equilibrium computer simulations we detect a non-monotonic behaviour of the melting line as a function of the frequency of the external field. This is attributed to a cage resonance effect which is controlled by the intrinsic polydispersity of the sample. The results are qualitatively explained within a cage theory of the polydisperse solid combined with a generalized Lindemann rule of melting.
\end{abstract}

\section{Introduction}

Recent experiments [1,2] have revealed that highly charged dusty particles in plasmas spontaneously form crystals due to their strong mutual repulsion. The structure, dynamics [3] and the melting scenario in quasi-equilibrium [4,5] and non-equilibrium [6] of these plasma crystals were studied in real space using direct imaging methods. Both quasi-two-dimensional sheets as well as three-dimensional bulk samples can be realized such that the effective dimensionality is tunable between two and three dimensions. The plasma crystal reveals a strong analogy to an ordinary colloidal crystal [7-9] of charged suspensions as the dusty particles are of mesoscopic size and the effective interactions are screened by the presence of microions. Furthermore, in both cases, the particles possess an intrinsic polydispersity in their charge which may also influence the phase behaviour as compared to a one-component (monodisperse) system [10-12]. There is, however, also an important difference between plasma and colloidal crystals. The colloidal crystal is typically embedded in a bath of a molecular thermal liquid while the dusty particles are surrounded by a plasma system. This implies that the dynamics of the mesoscopic particles is different: while it is completely overdamped by the solvent resulting in Brownian motion for the colloidal case, it is only slightly damped in the plasma case such that a Fokker-Planck description [13,14] of the individual particle dynamics is more appropriate [15].

In the present paper we study the melting transition of a plasma crystal under the influence of an time-dependent but space-independent external field applied to the bulk system. This is a non-equilibrium situation. Hence the melting scenario will depend on details of the particle dynamics. For a sinusoidal electric field, we find that the melting transition is shifted to lower temperatures with respect to the equilibrium case. This shift $\Delta T$ scales with the square of the relative polydispersity of the sample. Furthermore, as a function of the frequency of the alternating field we find resonance effects, i.e. the non-equilibrium melting line behaves nonmonotonically with increasing frequency. This is in striking contrast to the case of colloidal 
crystals with Brownian motion where no such resonances are expected [16]. We emphasize that this cage resonance effect is triggered and controlled by the intrinsic polydispersity. In particular, it disappears for monodisperse samples.

Most of out results are based on computer simulations using Fokker-Planck dynamics. We also propose a simple cage theory for the dynamics in the polydisperse crystal which we combine with a Lindemann melting rule applied to a non-equilibrium situation. We find qualitative agreement between theory and simulation.

Our paper is organized as follows. In section 2, the model is introduced and discussed. Then we describe the cage theory in section 3 . Our simulation techniques are summarized in section 4. Results are presented and discussed in section 5. We finally conclude with an outlook in section 6 .

\section{The model}

We consider $N$ dust particles in a volume $\Omega$ with a fixed number density $\rho=N / \Omega$ which sets the average interparticle spacing $a=\rho^{-1 / 3}$ as the typical length scale. The whole plasma crystal is held at fixed average temperature $T$. The dust particles $i$ and $j$ are interacting via an effective pair potential $V_{i j}(r)=Z_{i} Z_{j} U_{0} V_{0}(r)$ where $r$ is the interparticle distance and $U_{0}$ sets the energy scale. Here we have introduced a (dimensionless) polydispersity in the effective interaction between the dust particles by the intrinsic particle property $Z_{i}\left(Z_{i}>0\right)$. In fact, this particle property is a random variable $Z$ which is distributed according to a normalized distribution function $p(Z)$ with a mean value

$$
\bar{Z}=\int_{0}^{\infty} \mathrm{d} Z Z p(Z)
$$

and a relative polydispersity

$$
p_{Z}=\sqrt{\overline{Z^{2}} / \bar{Z}^{2}-1} \text {. }
$$

The leading term of the effective interaction $V_{0}(r)$ between the micrometre-sized grains is of Yukawa form [17] $V_{0}(r)=\exp (-\kappa r) / \kappa r$ with $\kappa$ denoting the inverse Debye screening length determined by the weakly coupled background plasma involving both ions and electrons. Radially symmetric ion-stream drag forces [18] may also be easily incorporated in our model. Effective dipole-dipole interactions [19] which may be induced by gravitational forces [18], however, require an anisotropic interaction which is beyond our level of description.

The dynamics of the dust particles is described in a Fokker-Planck picture with inertia, damping and collision terms [15]. Assuming that the mass $m$ and the friction constant $\gamma$ are the same for all particles, the stochastic Langevin equations for the particle trajectories $\vec{r}_{i}(t)$ $(i=1, \ldots, N)$ read as

$$
m \frac{\mathrm{d}^{2} \vec{r}_{i}}{\mathrm{~d} t^{2}}+\gamma \frac{\mathrm{d} \vec{r}_{i}}{\mathrm{~d} t}=-\vec{\nabla}_{\vec{r}_{i}} \sum_{j \neq i} V_{i j}\left(\left|\vec{r}_{i}-\vec{r}_{j}\right|\right)+\vec{F}_{i}(t)+\vec{F}_{i}^{(R)}(t) .
$$

The right-hand side of this equation involves three different forces: the interaction force, the force $\vec{F}_{i}(t)$ from the external oscillating field, and the random force $\vec{F}_{i}^{(R)}$ describing kicks of the microions. These kicks are Gaussian random numbers with zero mean, $\overline{\vec{F}}_{i}^{(R)}=0$, and variance

$$
\overline{\left(\vec{F}_{i}^{(R)}\right)_{\alpha}(t)\left(\vec{F}_{j}^{(R)}\right)_{\beta}\left(t^{\prime}\right)}=2 k_{B} T \gamma \delta_{\alpha \beta} \delta_{i j} \delta\left(t-t^{\prime}\right)
$$

according to the fluctuation-dissipation theorem. The subscripts $\alpha$ and $\beta$ stand for the three Cartesian components and $k_{B} T$ is the thermal energy. 
The space-independent external oscillatory force acting on the $i$ th particle points in the $z$-direction and is modelled as

$$
\vec{F}_{i}(t)=\vec{e}_{z} f_{i} \cos (\omega t)
$$

where $\omega$ is the external frequency, $\vec{e}_{z}$ is the unit vector along the $z$-direction and $f_{i}$ is the parameter describing the coupling of the $i$ th particle to the external field. The variables $\left\{f_{i}\right\}$ describe a second kind of polydispersity which we call coupling polydispersity. It is characterized by a normalized distribution function $p(f)$ with mean $\bar{f}$ and relative root variance

$$
p_{f}=\sqrt{\overline{f^{2}} / \bar{f}^{2}-1}
$$

Were the external field zero, then the problem would correspond to the dynamics in equilibrium and the time averages would follow Boltzmann statistics.

The Fokker-Planck dynamics has two limiting cases. For very small particle masses, the inertia term vanishes with respect to the damping and the dynamics reduces to the completely overdamped Brownian dynamics without hydrodynamic interactions as utilized for colloidal crystals in reference [16]. A possible measure for a typical timescale is

$$
\tau=\gamma a^{2} / U_{0}
$$

Using this timescale we can define a dimensionless parameter

$$
\alpha=m / \gamma \tau
$$

that controls the ratio between damping and inertia. The Brownian case of small inertia is then described by $\alpha \searrow 0$ at fixed $\tau$. The second limiting case occurs if the damping is very small, i.e. $\alpha \gg 1$. Then the dynamics is dominated by the inertia terms such that the equations of motion represent ballistic (Newtonian) dynamics in the limit $\alpha \rightarrow \infty$ at fixed $\tau$.

Let us now clarify why the coupling polydispersity is essential. In case of a monodisperse coupling ( $f_{i} \equiv f_{0}$ ), the result is pure equilibrium dynamics. This is easily seen by subtracting a global dynamical mode $\vec{r}_{0}(t)$ from the trajectories. The global mode is defined as a solution of the differential equation

$$
m \frac{\mathrm{d}^{2} \vec{r}_{0}}{\mathrm{~d} t^{2}}+\gamma \frac{\mathrm{d} \vec{r}_{0}}{\mathrm{~d} t}=\vec{e}_{z} f_{0} \cos (\omega t)
$$

The Langevin equations (3) can be rewritten in terms of new reduced trajectories $\tilde{\vec{r}}_{i}(t) \equiv$ $\vec{r}_{i}(t)-\vec{r}_{0}(t)$ such that the transformed equations have the same form as the original ones in the field-free case where $f_{i} \equiv 0$. Hence it is the coupling polydispersity that induces genuine non-equilibrium effects. Or in other words, in a monodisperse sample, the crystal melting line is not shifted by the presence of the external field.

\section{Fixed-cage theory}

In our theory we approximate the crystal as a harmonic solid using a cell approach with fixed neighbours located at the lattice positions $\left\{\vec{R}_{j}\right\}$. Such an approach was already applied to the completely overdamped Brownian dynamics in reference [16]. On average, the neighbour particles constitute a cage potential for which we assume no occupation correlations, i.e. we approximate in (3)

$$
\sum_{j \neq i} V_{i j}\left(\left|\vec{r}_{i}-\vec{r}_{j}\right|\right) \approx \sum_{j \neq i} \bar{Z} Z_{i} U_{0} V_{0}\left(\left|\vec{R}_{j}-\vec{r}_{i}\right|\right) \approx \bar{V}+\frac{1}{2} Z_{i} K\left(\vec{r}_{i}-\vec{R}_{i}\right)^{2}
$$


This requires that the polydispersity $p_{Z}$ is small. Moreover, we have assumed cubic crystal symmetry such that the harmonic picture becomes isotropic. $\bar{V}$ is an irrelevant additive constant and $K$ is an effective 'spring constant':

$$
K=\sum_{\vec{R}_{j} \neq 0} \frac{\bar{Z} U_{0}}{3}\left[V_{0}^{\prime \prime}\left(R_{j}\right)-2 V_{0}^{\prime}\left(R_{j}\right) / R_{j}\right] .
$$

Here one lattice point is at the origin; $V_{0}^{\prime}(r)$ and $V_{0}^{\prime \prime}(r)$ denote the first and second derivatives of $V_{0}$ with respect to $r$. Within these approximations, the stochastic Langevin equations describe a driven damped harmonic oscillator. Our goal is to calculate the averaged mean square displacement $\left\langle\vec{r}_{i}^{2}\right\rangle$ in the solid. The average involves both a polydispersity and a time average. One main assumption here is that the neighbours are fixed, i.e. they are only moving with the trivial overall dynamical mode $\vec{r}_{0}(t)$. Therefore we call this approach fixed-cage theory. The generalization of the result in reference [16] to Fokker-Planck dynamics is then

$$
\left\langle\vec{r}_{i}^{2}\right\rangle=\Delta_{1}+\Delta_{2}
$$

with the first term $\Delta_{1}$ arising from the equilibrium situation

$$
\Delta_{1}=\frac{3 k_{B} T}{K}\left\langle\frac{1}{Z_{i}}\right\rangle
$$

which reduces to

$$
\Delta_{1} \approx \frac{3 k_{B} T}{K \bar{Z}}\left(1+p_{Z}^{2}\right)
$$

for small interaction polydispersities. The second term $\Delta_{2}$ is an intrinsic non-equilibrium quantity generated by the external field:

$$
\Delta_{2}=\frac{\bar{f}^{2} p_{f}^{2}}{2} \frac{1}{m^{2}\left(\omega_{0}^{2}-\omega^{2}\right)^{2}+\gamma^{2} \omega^{2}}
$$

where

$$
\omega_{0}=\sqrt{K \bar{Z} / m}
$$

is the averaged eigenfrequency of the harmonic cage.

Let us now discuss this result: first of all, in the fully overdamped limit of very small masses $(\alpha \rightarrow 0)$, it reduces to the result of Brownian dynamics

$$
\Delta_{2}=\frac{\bar{f}^{2} p_{f}^{2}}{2} \frac{1}{(K \bar{Z})^{2}+\gamma^{2} \omega^{2}}
$$

studied earlier in reference [16]. The striking difference with respect to Fokker-Planck dynamics is the resonance embodied in the denominator of equation (14) which leads to a divergence in the ballistic limit of small damping $\alpha \rightarrow \infty$ at $\omega=\omega_{0}$. This can directly be attributed to a resonance of the dust particle in the harmonic cage formed by their neighbours which we call henceforth 'cage resonance'. Note that the whole cage is also oscillating under the influence of the field; it is only the relative motion of a particle with a coupling parameter different from the average $\bar{f}$ that leads to the resonance effect. Consequently polydispersity is one relevant parameter for observing cage resonance, i.e. $\Delta_{2}=0$ for $p_{f}=0$. Hence our cage resonance effect is different from ordinary phonon resonances which occur also in monodisperse samples. 
To access the solid melting curve we now use a generalized Lindemann rule [16, 20]. According to this phenomenological criterion, a solid melts in equilibrium if the Lindemann parameter defined as

$$
L=\frac{\sqrt{\left\langle\vec{r}_{i}^{2}\right\rangle}}{a}
$$

is roughly 15 per cent. Postulating that this rule is also valid in our non-equilibrium situation, the solid melting curve is obtained by

$$
\frac{\sqrt{\left\langle\vec{r}_{i}^{2}\right\rangle}}{a}=L_{0}
$$

where $L_{0}$ is the Lindemann parameter for the associated monodisperse system in equilibrium. This relation together with equation (11) determines the location of the solid melting. We remark that the basic assumption of the Lindemann criterion in non-equilibrium can be separately checked by non-equilibrium computer simulations. In fact we shall do this in the remainder of this work, finding reasonable justification for using a generalized Lindemann rule from our simulation data.

Summarizing, our theory predicts for the shift $\Delta T \equiv T_{0}-T$ of the melting temperature (with $T_{0}$ denoting the melting temperature in equilibrium)

$$
\Delta T=\frac{m \omega_{0}^{2} \bar{f}^{2} p_{f}^{2}}{6 k_{B}\left(1+p_{Z}^{2}\right)} \frac{1}{m^{2}\left(\omega_{0}^{2}-\omega^{2}\right)^{2}+\gamma^{2} \omega^{2}} .
$$

The melting temperature shift $\Delta T$ has a maximum for the frequency

$$
\omega_{c}=\sqrt{\omega_{0}^{2}-\frac{\gamma^{2}}{2 m^{2}}}
$$

with a typical full half-width $\Delta \omega$. For small damping $\gamma, \omega_{c}=\omega_{0}$ and $\Delta \omega=\gamma / m$. One important prediction of the theory is that $\Delta T$ scales with the square of the relative coupling polydispersity for small polydispersities. Clearly, our theory is only valid for small polydispersities. For large coupling polydispersities, the melting temperature becomes formally negative as $\Delta T>T_{0}$. This is due to the fact that the external field produces a Lindemann parameter which is larger than $L_{0}$ even at $T=0$, such that the system is already molten at zero temperature.

\section{Fokker-Planck dynamics computer simulations in non-equilibrium}

Our code is very similar to an equilibrium simulation using Fokker-Planck dynamics [21]. We put $N=864$ particles into a cubic box of length $\ell$ with periodic boundary conditions. The colloidal number density is $\rho=N / \ell^{3}$. The particles interact via an effective Yukawa pair potential

$$
V_{i j}(r)=Z_{i} Z_{j} U_{0} \exp (-\kappa r) / \kappa r .
$$

The dimensionless charges $Z_{i}$ are drawn from a rectangular distribution around a mean value $\bar{Z} \equiv 1$ with a prescribed polydispersity $p_{Z}$. The coupling polydispersity is taken to be proportional to the charge polydispersity, i.e. $f_{i}=E_{0} Z_{i}$ where $E_{0}$ measures the amplitude of the oscillating electric field. The direction of the external force is always along an edge of the simulation box. We remark that in equilibrium, any thermodynamic quantity only depends on the two parameters $\lambda=\kappa \rho^{-1 / 3} \equiv \kappa a$ and $T^{*}=k_{B} T \lambda \exp (\lambda) / U_{0}$ [22]. The dynamics in equilibrium depends also on $\alpha=m / \gamma \tau$, determining the ratio of inertia and damping. In 
our runs, we either fix $\alpha$ to 90.7 to describe a slightly damped Fokker-Planck motion or we set $\alpha=0$ in order to establish a direct comparison with pure Brownian motion. The choice $\alpha=90.7$ is arbitrary. As a function of $\alpha$ one expects a smooth crossover from the completely overdamped Brownian case $(\alpha=0)$ to the Newtonian case such that one particular choice of $\alpha \gg 1$ is sufficient in order to get the qualitative features of the non-equilibrium melting in the case where inertia terms dominate the dynamics. In a non-equilibrium steady state, with an alternating external field, there are two further parameters, namely the field amplitude $E_{0}$ and its frequency $\omega$. While we vary the latter in our simulations, we keep the amplitude $E_{0}$ fixed to $E_{0}=0.0055 U_{0} / a$

Our starting configuration was a face-centred-cubic (fcc) lattice which was randomly occupied by particles of different charge. For $\lambda=5.8$, as chosen throughout our simulations, this structure is the thermodynamically stable one, at least for small polydispersities [22]. We have averaged our results over different random occupations and checked that they were not affected by the initial occupation. The Langevin equations of motion including the shaking external field were numerically solved using a finite time step $\Delta t$ and the technique of Ermak and Buchholz [23]. The typical size of the time step was $\Delta t=0.008 \tau$.

We studied the system as a function of temperature $T^{*}$, starting with small temperatures and continuing our simulations by heating the system discontinuously in small steps of typically $\delta T^{*}=0.01$. Typically $6 \times 10^{4}$ time steps in each elementary heating process were simulated which corresponds to a simulation time of $480 \tau$. After an initial relaxation period of $240 \tau$, statistics were gathered. During the simulations, we monitored the Lindemann parameter $L$ of the particles, the pair distribution function and the bond orientational correlation function [24] in order to locate the crystal melting transition. The system either runs to a steady-state situation where the fcc solid remains stable or it suddenly melts, losing its crystalline order. The melting process is signalled by a drastic increase of the Lindemann parameter. Cooling the system down again results in a hysteresis loop for the average mean square displacement. The width of this loop is an estimate for the melting point both in equilibrium and in non-equilibrium.

\section{Results}

In figure 1 , computer simulation results for the Lindemann parameter $L$ are shown versus reduced temperature $T^{*}$. Upon heating the initial crystal, $L$ is increasing slightly with temperature until the melting point is reached. Here $L$ starts to increase significantly, signalling that the resulting steady state is a fluid. Cooling the fluid down again leads to a hysteresis loop also shown in figure 1 . The position of the hysteresis fixes the crystal melting temperature. It is demonstrated in figure 1 that, for a non-equilibrium situation with an external field, the same diagnostics can be applied to obtain the melting temperature as in equilibrium. The width of the hysteresis loop becomes even smaller in non-equilibrium.

One of the basic assumptions in our fixed-cage theory was the constancy of the Lindemann parameter at melting in non-equilibrium. This assumption is tested in figure 2. Here again $L$ is plotted versus temperature in non-equilibrium for different frequencies. From the plot it can indeed be deduced that the rapid increase of $L$ signalling crystal melting occurs when $L$ is roughly constant, $L \approx 0.2$. For comparison, we also show the equilibrium situation where $L_{0}=0.17$. Hence the Lindemann criterion can be transferred to a non-equilibrium situation. From figure 2, it can already be concluded that the melting temperature behaves non-monotonically with frequency $\omega$. This will be discussed in more detail later.

In figure 3 , we show the structure of the steady-state non-equilibrium fluid. The radial distribution function is anisotropic as the external field destroys radial symmetry. We define 


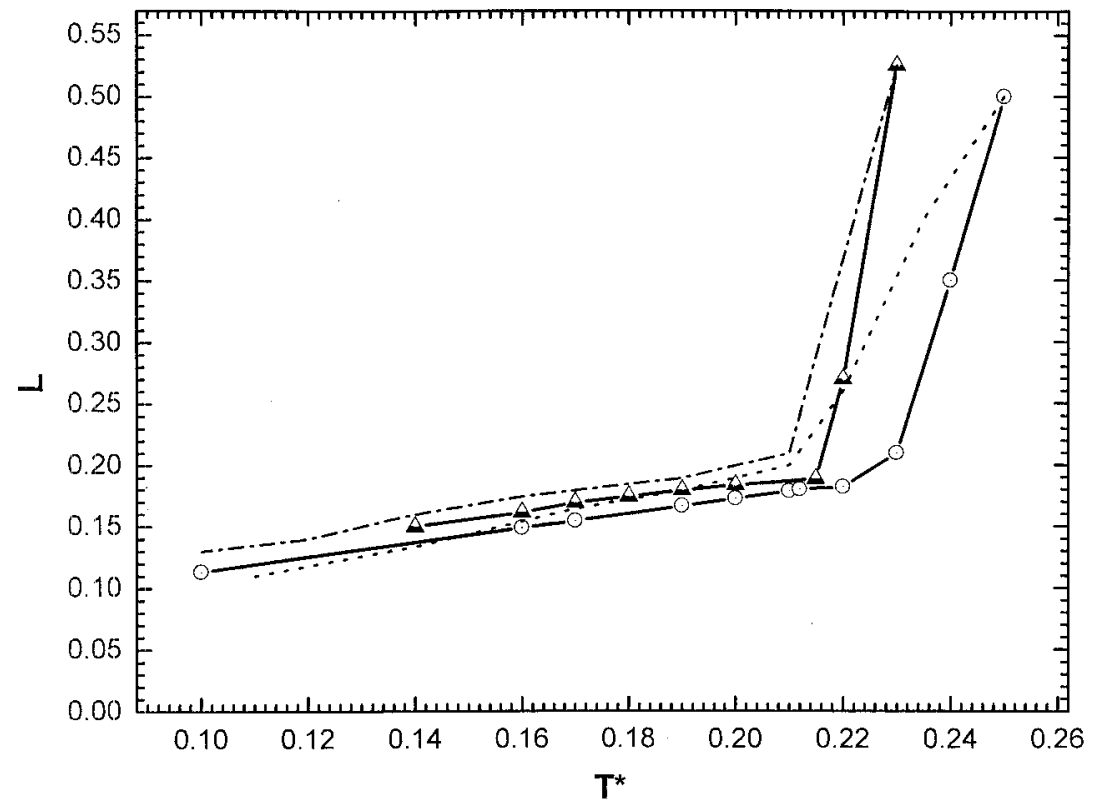

Figure 1. Computer simulation results for the averaged Lindemann parameter $L$ versus reduced temperature $T^{*}$ along a heating and cooling cycle. The polydispersity is $p_{Z}=0.02$ and a value of $\alpha=90.7$ was used. The circles are for a heating process in an equilibrium situation without external field while the dotted curve is for the corresponding cooling process. The triangles and the dash-dotted line are for a heating and cooling cycle in a non-equilibrium situation with a field frequency of $\omega \tau=0.785$.

the spherical averaged pair distribution function via

$$
g(r)=\frac{1}{4 \pi \rho N} \int \mathrm{d}^{3} r^{\prime} \delta\left(r-r^{\prime}\right)\left\langle\sum_{i, j=1, i \neq j}^{N} \delta\left(\vec{r}^{\prime}-\left(\vec{r}_{i}-\vec{r}_{j}\right)\right)\right\rangle .
$$

The function $g(r)$ exhibits a similar fluid structure as in equilibrium which becomes evident from figure 3(a). In fact, comparing an equilibrium versus non-equilibrium situation while keeping all other parameters unchanged, the different $g(r)$ s practically coincide. This implies that the presence of the shaking field has no significant influence on the fluid pair structure. We remark that there is no theory available to predict this structure in non-equilibrium as liquid integral equation theory is constructed only for equilibrium cases [25]. The averaged pair distribution $g(r)$ is different in the solid state where it exhibits long-ranged correlations visible as peaks in $g(r)$ even for large distances. If one compares equilibrium versus non-equilibrium in the solid phases, there is a large loss in the solid structure induced by the external field; see figure 3(b). This is in striking contrast to the case for the fluid phase where the external field does not affect the correlations. The physical reason for this is that the cage of neighbours is rigid in the solid but mobile in the fluid.

The key plot showing the cage resonance effect is contained in figure 4 . Here the melting temperature is shown versus the frequency $\omega$ of the external field for different polydispersities. In figure 4(a), Fokker-Planck dynamics is used ( $\alpha=90.7)$. In this case the melting temperature behaves non-monotonically with frequency and exhibits a minimum at $\omega=\omega_{c}$. This clearly demonstrates the cage resonance effect. The sharpness of the resonance can be described by a typical full half-width $\Delta \omega$ in frequency around $\omega=\omega_{c}$. For pure overdamped Brownian 


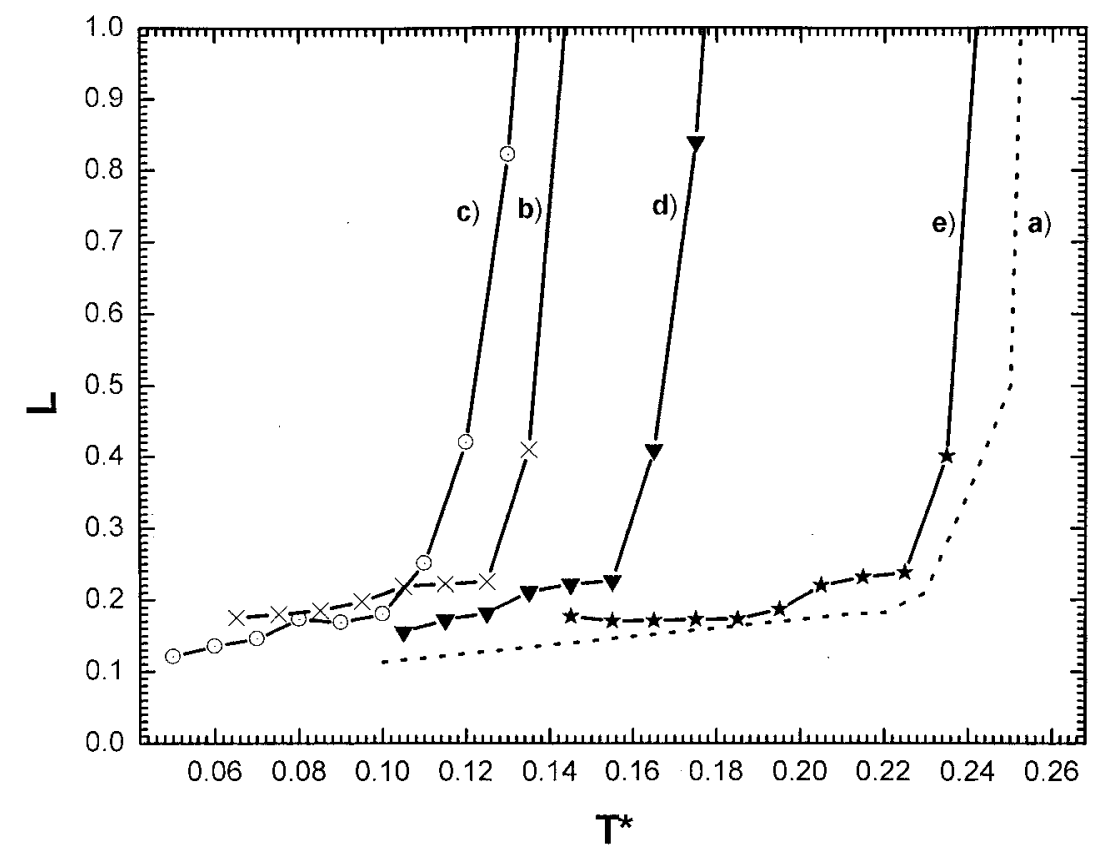

Figure 2. As figure 1, but now used as a test of the Lindemann criterion. For different frequencies of the external field the heating curves are shown. The rapid increase of the Lindemann parameter occurs at different temperatures but always for $L \approx 0.2$. The dotted curve (a) shows the equilibrium situation. The frequencies are (b) $\omega \tau=0.45$, (c) $\omega \tau=0.8$, (d) $\omega \tau=1.25$, (e) $\omega \tau=1.571$. Further parameters are $p_{Z}=0.05$ and $\alpha=90.7$.

dynamics, on the other hand, figure 4(b) shows that there is no resonance effect at all—which is thus a genuine feature induced by the inertia term in the equation of motion.

We now compare the simulation results to those of fixed-cage theory. First of all, the actual numbers for the polydispersity are too large for frequencies near resonance to guarantee positive melting temperatures. Still, the position of the maximal temperature shift at $\omega_{c}$ agrees well with the theoretical one, coinciding with the eigenfrequency of the cage. The half-width $\Delta \omega$, however, is much larger than that predicted from theory. This is due to the fact that in the actual solid there is a distribution of cage resonances at eigenfrequencies different from $\omega_{0}$ resulting from the finite polydispersity in the solid.

In figure 5 , we test the theoretical prediction that the temperature shift $\Delta T$ scales with the square of the relative polydispersity. For that purpose, we plot the reduced temperature shift $\Delta T^{*} / p_{Z}^{2}$ versus frequency $\omega$. If the scaling holds, all data points should collapse onto a single curve. In fact, within the error bars of the simulation, this is confirmed by figure 5 . In particular, $\omega_{c}$ and $\Delta \omega$ are independent of polydispersity. The theoretical curve exhibits a very sharp peak at $\omega \tau=0.821$ with a large height of $\Delta T^{*} / p_{Z}^{2}=1369$ which we have not plotted for clarity. Again, while the position of the peak is in good agreement with the simulation, its amplitude is too large and its width too small.

\section{Conclusions}

We have investigated the Fokker-Planck dynamics of the non-equilibrium melting transition of a polydisperse crystal in an external oscillatory field. We detected a cage resonance effect 

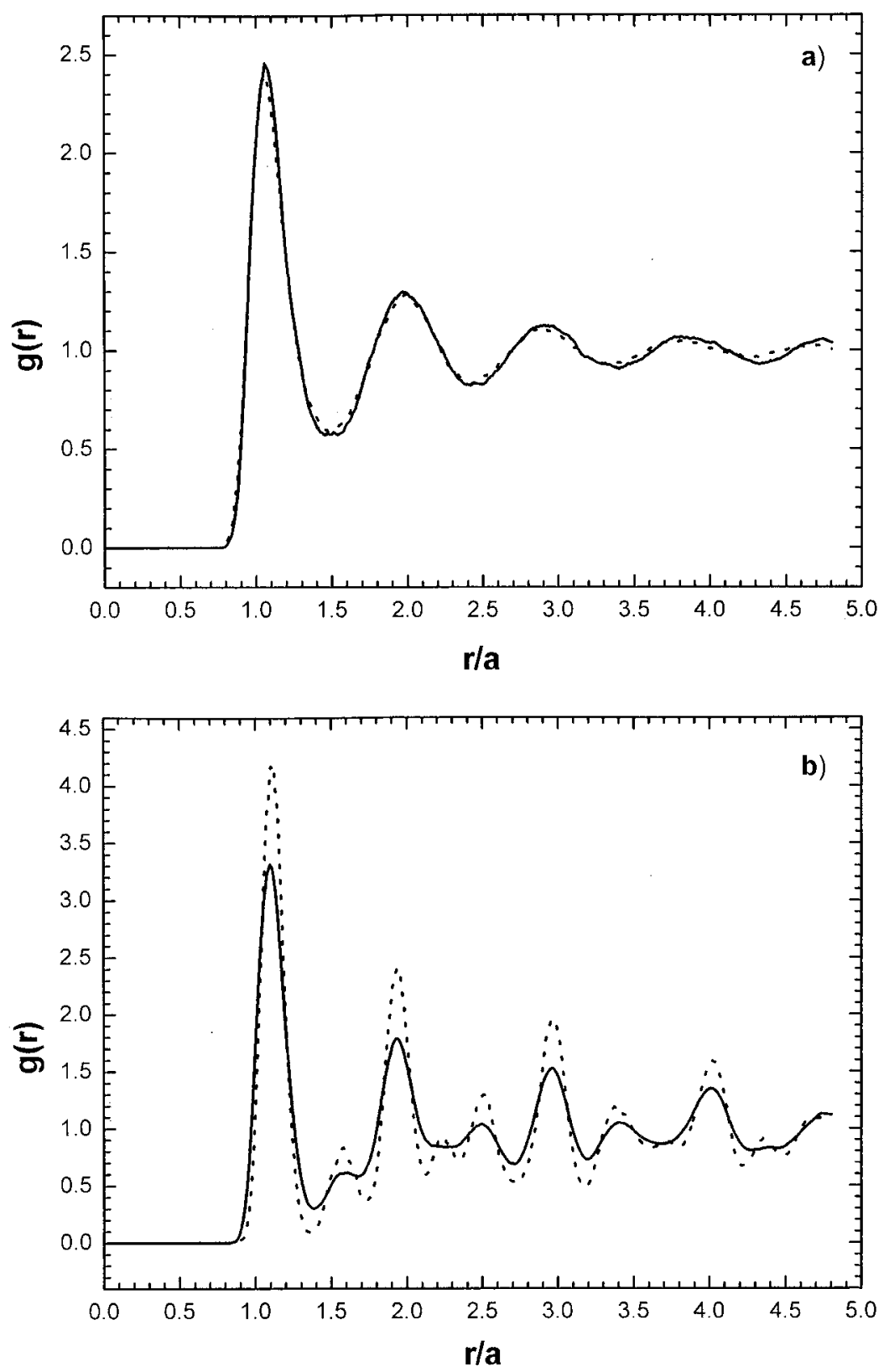

Figure 3. Radially averaged pair distribution function $g(r)$ versus reduced distance $r / a$. The polydispersity is $p_{Z}=0.02$ and a value of $\alpha=90.7$ was used. (a) The fluid state at temperature $T^{*}=0.25$; the dotted curve is for equilibrium while the solid curve is for a field frequency $\omega \tau=0.785$. (b) As (a), but now for a solid state at temperature $T^{*}=0.11$.

which vanishes for overdamped motion and for monodisperse samples. A simple theory attributes the resonance to the collective motion of the cage formed by neighbouring solid particles. The theory was qualitatively confirmed by non-equilibrium computer simulations in non-equilibrium. 

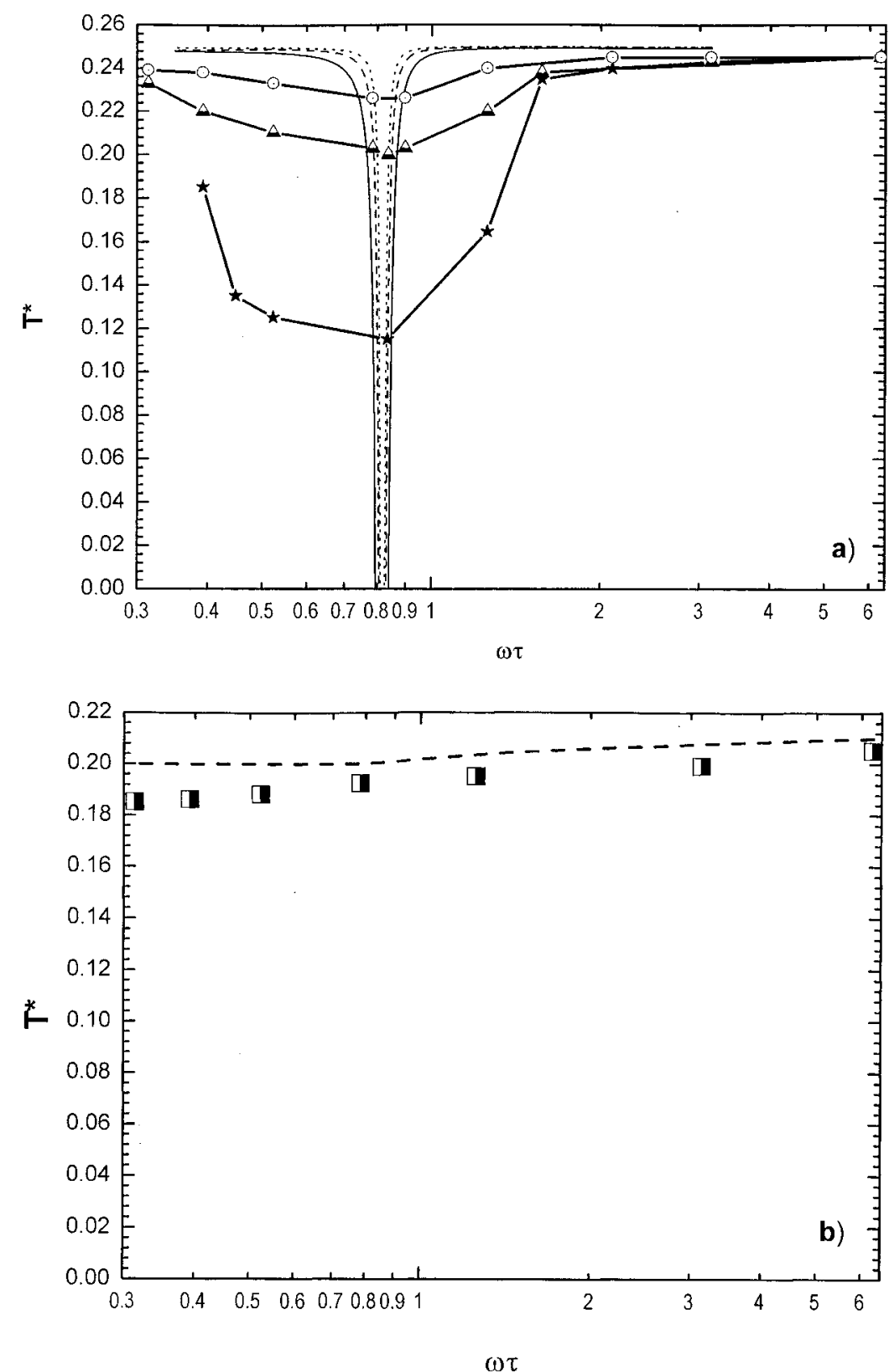

Figure 4. The melting temperature $T^{*}$ away from equilibrium under the influence of an oscillating field as a function of the reduced field frequency for different relative charge polydispersities $p_{Z}=0.02,0.03,0.05$ (from top to bottom). Note that the frequency scale is logarithmic. (a) Fokker-Planck dynamics $(\alpha=90.7)$ : symbols denote simulation data; curves are obtained from fixed-cage theory. Note that in the theory the melting temperature is zero around the resonance. (b) As (a), but now for Brownian dynamics $(\alpha=0)$.

We emphasize that our model for the dusty plasma is simple, which has both a disadvantage and an advantage: the disadvantage is that some important circumstances which can become 


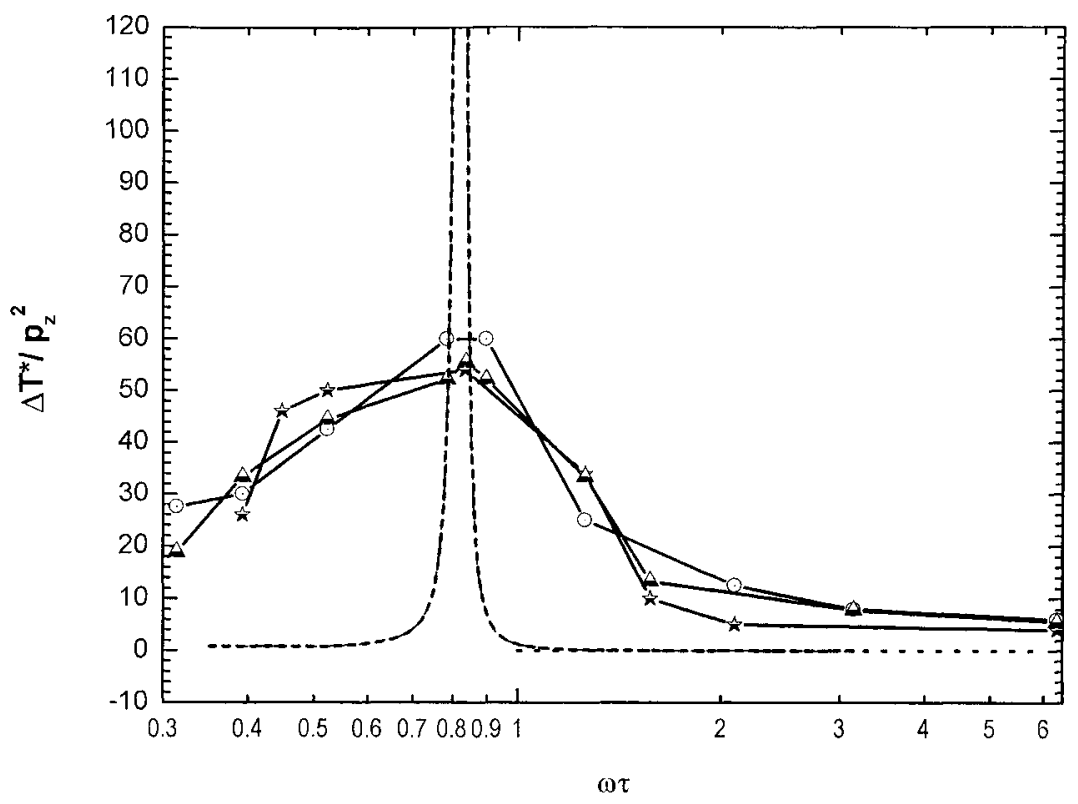

Figure 5. Reduced temperature shift $\Delta T^{*} / p_{Z}^{2}$ versus frequency $\omega$ for the simulation data (symbols) and the theory (broken line) of figure $4(a)$.

relevant for real samples of dusty plasma crystals are not incorporated, e.g. an underlying ion stream. The advantage, on the other hand, is that genuine results of our simple model may be verified for samples different from dusty plasmas. These include strongly interacting charged aerosol grains [26] in a crystalline state, ferromagnetic colloidal particles in an oscillating magnetic field [27] and periodically shaken arrays [28] of neutral granular matter [29]. Therefore our theoretical predictions can in principle be verified in experiments on dusty plasmas as well as ones on granular materials.

We finish with a few remarks.

First, we have assumed the same mass and friction coefficient for every particle. In principle, a mass and friction polydispersity can also be incorporated in the theoretical description. The fixed-cage model, for instance, can be readily generalized in this direction leading to a similar cage resonance effect. For non-vanishing relative mass polydispersity $p_{m}$, for example, the prefactor $p_{f}^{2}$ in equation (14) has to be replaced by $p_{f}^{2}+p_{m}^{2}$, showing that mass polydispersity leads to cage resonance even if the coupling polydispersity $p_{f}$ is zero.

Second, there is a need for a quasi-equilibrium theory in the steady-state situations.

Third, the fixed-cage assumption can be avoided in a more sophisticated treatment of the solid. Relying still on a harmonic description, this requires the full diagonalization of the dynamic matrix in the equations of motion.

\section{Acknowledgments}

We thank M Schmidt and A Zippelius for helpful remarks. Financial support from the Deutsche Forschungsgemeinschaft within the Graduiertenkolleg Hochtemperaturplasmaphysik is gratefully acknowledged. 


\section{References}

[1] Thomas H M, Morfill G E, Demmel V, Goree J, Feuerbacher B and Möhlmann D 1994 Phys. Rev. Lett. 73652

[2] Morfill G E and Thomas H M 1996 J. Vac. Sci. Technol. A 14490

[3] Zuzic M, Thomas H M and Morfill G E 1996 J. Vac. Sci. Technol. A 14496

Schweigert V A, Schweigert I V, Melzer A, Homann A and Piel A 1998 Phys. Rev. Lett. 805345

[4] Thomas H M and Morfill G E 1996 Nature 379806

[5] Thomas H M and Morfill G E 1996 J. Vac. Sci. Technol. A 14501

[6] Schweigert V A, Schweigert I V, Melzer A, Homann A and Piel A 1998 Phys. Rev. Lett. 805345

[7] For a review, see Pusey P N 1991 Liquids, Freezing and the Glass Transition ed J-P Hansen, D Levesque and J Zinn-Justin (Amsterdam: North-Holland)

[8] Arora A K and Tata B V R 1998 Adv. Colloid Interface Sci. 7849

[9] Löwen H 1994 Phys. Rep. 237249

[10] Löwen H, Roux J N and Hansen J-P 1991 Phys. Rev. A 441169

Löwen H, Hansen J-P and Roux J N 1991 J. Phys.: Condens. Matter 3997

[11] D’Aguanno B and Klein R 1991 J. Chem. Soc. Faraday Trans. 87379

D'Aguanno B and Klein R 1992 Phys. Rev. A 467652

[12] Tata B V R and Arora A K 1991 J. Phys.: Condens. Matter 37983

[13] Fokker A D 1914 Ann. Phys., Lpz. 43810

[14] Planck M 1917 Sitzber. Preuss. Akad. Wiss. p 324

[15] Risken H 1989 The Fokker-Planck Equation 2nd edn (Berlin: Springer)

[16] Löwen H and Hoffmann G P 1999 Phys. Rev. E 603009

[17] Morfill G E, Thomas H M, Konopka U and Zuzic M 1999 Phys. Plasmas 61769

[18] Lee H C and Rosenstein B 1997 Phys. Rev. E 557805

[19] Mahmoodi J, Shukla P K, Tsintsadze N L and Tskhakaya D D 2000 Phys. Rev. Lett. 842626

[20] Lindemann F A 1910 Phys. Z. 11609

[21] See, e.g., Gleim T, Kob W and Binder K 1998 Phys. Rev. Lett. 814404

[22] Meijer E J and Frenkel D 1991 J. Chem. Phys. 942269

Stevens M J and Robbins M O 1993 J. Chem. Phys. 982319

[23] Ermak D L and Buchholz H 1980 J. Comput. Phys. 35169

[24] van Duijneveldt J S and Frenkel D 1998 J. Chem. Phys. 964655

[25] See, e.g., Hansen J-P and McDonald I R 1986 Theory of Simple Liquids 2nd edn (London: Academic)

[26] Wolf D E, Scheffler T and Schäfer J 1999 Physica A 274171

[27] Mahr T and Rehberg I 1998 Physica D 111335

[28] Scherer M A, Mahr T, Engel A and Rehberg I 1998 Phys. Rev. E 586061

[29] Ristow G H, Strassburger G and Rehberg I 1997 Phys. Rev. Lett. 79833 